\title{
A Method for Teaching the Modeling of Manikins Suitable for Third-Person 3-D Virtual Worlds and Games
}

\author{
Nick V. Flor* \\ Information Systems, Film, \& Digital Media, Anderson School of Management, University of New Mexico
}

\section{Abstract}

Virtual Worlds have the potential to transform the way people learn, work, and play. With the emerging fields of service science and design science, professors and students at universities are in a unique position to lead the research and development of innovative and value-adding virtual worlds. However, a key barrier in the development of virtual worlds - especially for business, technical, and non-artistic students-is the ability to model human figures in 3-D for use as avatars and automated characters in virtual worlds. There are no articles in either research or teaching journals which describe methods that non-artists can use to create 3-D human figures. This paper presents a repeatable and flexible method I have taught successfully to both artists and business students, which allows them to quickly model human-like figures (manikins) that are sufficient for prototype purposes and that allows students and researchers alike to explore the development of new kinds of virtual worlds.

Keywords: Teaching, Multi-User Virtual Environments, Learning.

Received on 9 September 2011; accepted on 16 April 2012; published on 10 August 2012

Copyright (C) 2011 Flor, licensed to ICST. This is an open access article distributed under the terms of the Creative Commons Attribution licence (http://creativecommons.org/licenses/by/3.0/), which permits unlimited use, distribution and reproduction in any medium so long as the original work is properly cited.

doi: 10.4108/eeel.2012.07-09.e2

\section{Introduction}

The widespread incorporation of advanced graphics processors in cellphones, tablets, and other mobile devices-combined with the availability of portable fullbody motion capture controllers such as the Microsoft Kinect ${ }^{\mathrm{TM}}$ - is creating a kind of digital ecosystem in which 3-D Virtual Worlds can operate.

A 3-D virtual world is a computer program that displays to users 3-D representations of people, places, and other things found in the "real world," and that allows users to flexibly interact with these representations via standard input devices like a keyboard and mouse, or more sophisticated input devices like accelerometers or motion capture controllers.

*Corresponding author. Email: nickflor@unm.edu
A third-person 3-D virtual world also contains an avatar-a character under a user's control that the user can see and that is central to the user's feeling of immersiveness in the virtual world (see Figure 1). Avatars do not have to look anything like a user, nor do they have to look human, but human-like figures are the most common type of avatars in virtual worlds.

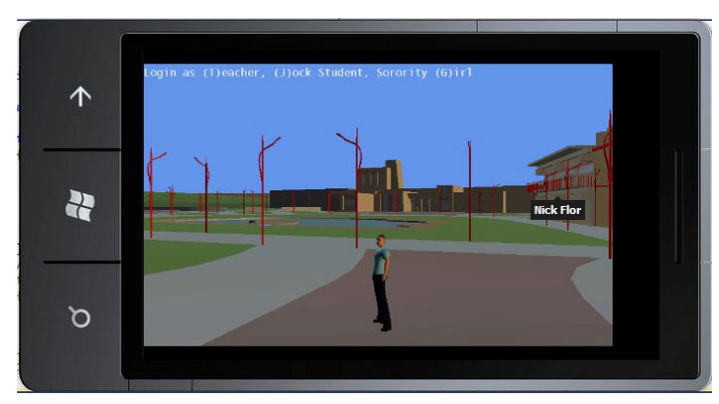

Figure 1. Virtual UNM Campus Developed by the Author on a Mobile Device, Avatar in the Center 
Similar to when the web first emerged as a technology, the most innovative uses of virtual worlds lie waiting to be discovered. If university researchers and students are to lead the discovery of innovations in virtual worlds it is necessary for them to have the ability to build and experiment with different kinds of virtual worlds, rather than work under the constraints of existing commercial virtual worlds. A key barrier to building virtual worlds is modeling avatars.

\section{LITERATURE REVIEW ON 3-D HUMAN FIGURE MODELING}

The value of virtual worlds as educational tools has been noted prior to the turn of the century (Dede, 1996). Researchers are starting to explore the potential of virtual worlds as tools for studying social behaviors scientifically (Bainbridge, 2007), for education (Vincenti \& Braman, 2010) for business-related purposes (Hemp, 2006). How to build virtual worlds has not been a primary topic of research.

Most of the research on business uses of virtual worlds has focused on analyzing how companies use existing, commercially available virtual worlds, such as Second Life or World of Warcraft. Missing in the academic literature is research on how to actually build new kinds of virtual worlds, as this is viewed as practice not research. Instead, such knowledge is distributed throughout trade books written by either art professors (Ratner, 2009) or by practitioners (Patnode, 2008).

However, research on how to build new systems is important too. The emerging fields of Design Science (Hevner, March, Park, \& Ram, 2004) and Service Science (Maglio \& Spohrer, 2008), argue that as a science of the artificial (Simon, 1996) it is just as important for researchers to create new, value-adding artifacts as it is to study existing ones.

The technology for building virtual worlds has advanced to the point where a single individual unaided can build one (Flor, 2011). A key enabling skill for implementing virtual worlds is modeling human figures. Although the aforementioned trade books all discuss methods for modeling human figures, these books are written for art students and most of the techniques are not repeatable by non-artists and are more suited for games and movies than virtual worlds.

The method presented below is one that non-artists can follow to prototype a human figure (a "manikin") and that can be easily modified to create different types of avatars. It qualifies as design-science research under the seven guidelines of design science (Hevner, March, Park, \& Ram, 2004, p. 82). In particular the method: (1) is a useful and novel artifact; (2) has a specific problem domain-virtual worlds; (3) has been tested on nonartists; (4) allows non-artists to perform a difficult task, namely modeling 3-D human figures; (5) is defined rigorously so that non-artists can duplicate the steps needed to create a 3-D figure; (6) was developed after analyzing the difficulties in the space of existing modeling methods. The seventh guideline is to communicate the results effectively to technical and managerial audiences, which is the goal of this paper.

Before discussing the method in this paper for modeling 3-D human figures, we will first review how professional modelers in the movie and gaming industries typically model characters.

\section{3-D Tracing}

The method most commonly used in industry to model human figures is commonly referred to as character modeling with reference pictures, although I use the term "3-D tracing" because it is analogous to the technique of placing see-through paper over a photograph and creating a drawing by tracing. The method can be seen in a variety of books including, Guindon (2007, pp. 15-83), Ingrassia (2009, pp. 269-288), Oliverio (2007, pp. 47-323), Patnode (2008, pp. 133-207), and Russo, (2006, pp. 83-152). The major steps are as follows (see Figure 2 for depictions of the steps):

1. The modeler first obtains reference pictures. In the movie and videogaming industries, these reference pictures are typically drawn by a concept artist in the art department. However, if the 3-D model is intended to represent an actual person, then reference photographs of the actual person are used instead of drawings. The reference pictures depict front and side views of the character to be modeled, with features lined up in both photos including the top of the head, the waistline, and the bottom of the feet.

2. The reference pictures are then imported into a modeling program. The pictures are placed at right angles to one another, and centered.

3. The modeler adds a simple box to the workspace so that it overlaps the torso in both the front and the side reference-picture views.

4. Finally, the modeler molds the box to the reference drawings - extruding limbs, adding edge loops, and moving vertices so that the edges of the model line up with the edges in the reference pictures. 


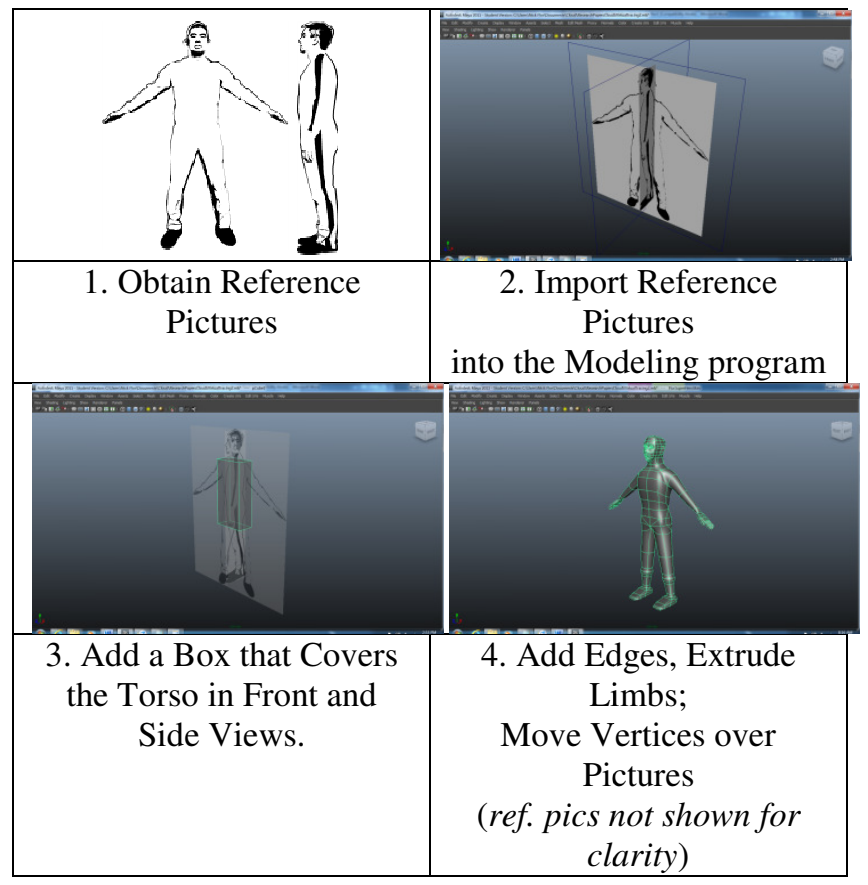

Figure 2. 3-D Tracing: A Modeler (1) Takes front and side pictures of a human figure; (2) Imports them into a 3-D modeling program; (3) Adds a basic box that covers the torso; (4) Mold the box to the pictures.

3-D tracing is by far the most common technique for modeling human figures. Yet another more advanced method for figure modeling is digital sculpting (de la Flor \& Mongeon, 2010; Spencer, 2010), where the modeler creates an entire character without reference pictures. However, digital sculpting requires detailed knowledge of body proportions and surface anatomy.

\section{Comparison of 3-D Tracing with my Manikin Modeling Method}

Methods for modeling human figures in 3-D are certainly not new. Moreover, there are an abundance of tutorials on the "3-D tracing" method written by both professional modelers and by modeling enthusiasts, in trade books and in social-media video websites like YouTube.

There are three main problems with 3-D tracing as a method for non-artists. First, it requires reference pictures. If the modeler is not an artist, then development is hindered while the modeler either works with an artist, or finds pictures, or takes photographs. Taking photographs can be non-trivial, as reference pictures may require actors, costumes, and props to be built. Second, it is time consuming to model using 3-D tracing as the modeler must constantly move vertices and edges to line them up with the reference pictures in both front and side views. In fact, one professional video game modeler reported taking on average one to three weeks to complete a figure (Olson, 2009), although it should be noted that these were detailed figures. Third, detailed human figures created via 3-D tracing are unnecessary for prototyping useful virtual worlds, which is the main goal of virtual world experimenters.

The manikin method has the following benefits versus the 3-D tracing method. First, it is easy to teach to nonartists. I have taught the method in a 1.5 hour lecture to business students, none of which were artists. Second, the method is easy to learn and its application produces repeatable results. Over ninety percent of business students who were taught the method, successfully modeled a 3-D manikin in under a week. Third, the method is flexible and extendable as different kinds of manikins can be created, and detailed can be added to these manikins at a later time if needed, using a variation of the 3-D tracing method. Finally, the method creates human figures that are good enough for prototyping purposes. In my courses, the usefulness of the virtual worlds that the students create takes precedence over aesthetics. By not having to spend so much time on modeling characters, students can focus on functionality and add aesthetics to their manikins at a later time. This is similar to the approach taken in figure drawing, where artists first draw a posed human figure using cylinders, cubes, spheres and other basic geometric primitives (Loomis, 1943). This allows the artist to focus on both the action depicted and the meaning of the action-details like muscles, clothing, and shadow are added later.

In the following sections, we examine the manikin method and the test of the method on both artistic and non-artistic students.

\section{A REPEATABLE AND FLEXIBLE METHOD FOR MODELING HUMAN FIGURES IN 3-D: THE MANIKIN METHOD}

3-D modeling is an activity where an individual creates representations of real or imagined things - that appear to have width, height, and depth — using a special kind of computer program known as modeling software. The representations that one creates using modeling software are called models, and the person that creates them is a modeler

\section{Choosing Modeling Software-Freely- Available Packages}

Popular commercial modeling software packages include Maya and 3ds-Max, both by Autodesk Corporation, while the most popular open-source modeling software package is Blender. Both Maya and 3ds Max are expensive software packages, as they are used to create models for both Hollywood movies and videogames. However, 
Autodesk makes them available freely, with full functionality; the only restriction is that anything created by the software is for academic-use only. Instructors and students can download these packages from students.autodesk.com, after registering with an e-mail address from a recognized academic institution. Blender can be downloaded from www.blender.org.

Most all 3-D modeling software packages are sufficient for modeling human figures using the manikin method. The decision of what package to use in a course will depend largely on the computers available to the instructor and to the students - as not all modeling software will run on all operating systems. In particular, both Maya and Blender run on the Windows, the Mac, and the Linux operating systems. 3ds Max runs only on the Windows operating system. At the University of New Mexico's Interdisciplinary Film \& Digital Media program, Maya is the preferred package since the students own both Macs and PCs, and it is the modeling software most commonly used by the movie industry, which is a target employer for the students.

\section{Polygon Modeling}

There are two basic ways to model, using polygons or using curves. This paper covers polygon modeling-the most popular kind of modeling for virtual worlds. The person that creates the 3-D models is known as a modeler. Using polygon modeling, the modeler starts with a basic object - e.g., a cube, sphere, or pyramid with a certain width $\mathrm{x}$ height $\mathrm{x}$ depth and with a certain number of faces - much like a sculptor starts with a lump of clay. The modeler then molds this source object into a target object using the operations of moving, cutting, extruding, wedging, merging, deleting, and mirroring, which are applied to the object's vertices, edges, and faces.

The following describes the general lecture script that I developed for teaching students how to model a 3-D manikin. I have intentionally written the lecture script in general terms-without reference to any software-specific operation-so that an instructor can adapt it easily to the specific modeling software that he or she uses. The script assumes the instructor and students have working knowledge of the basic modeling operations, which are listed in italics in the script. For those unfamiliar with basic modeling operations, Appendix A provides a tutorial of these operations specific to the Maya software package.

\section{Part 1. Creating the Body}

To create a body: (1) Create a cube that is $1 \times 2.5 \times 1$ units with $2 \times 5 \times 2$ faces; (2) Wedge the top back face 45 degrees, yielding an arm face; (3) Move the outerbottommost edges +.1 units in the $\mathrm{x}$ direction yielding a leg face; (4) Extrude the arm face 2.5 units, inset by .1 units; (5) Extrude the leg-face 2 units yielding a thigh; (6) Move the thigh face's inner-bottommost edge +.1 units in the x-direction; (7) Extrude the thigh face 2 units, inset by .1 units, yielding a calf; (8) Move the middle back vertices to a $\mathrm{z}$-value of zero and merge the overlapping vertices, yielding a back wedge; (9) Mirror to see what the completed body will look like; (10) Save file as "body".

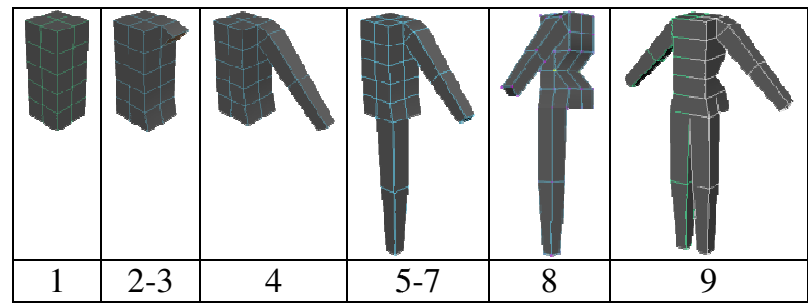

Figure 3. Modeling the Body (see text for explanation)

\section{Part 2. Creating the Hand}

To create a hand: (1) Create a cube $.5 x .125 \times .5$ with $2 \times 1 \times 4$ faces; (2) Wedge the thumb face 30 degrees; (3) Inset the stubs .1; (4) Extrude the fingers .5 with 3 divisions; (5) Extrude the thumb .25, then extrude again by .125; (6) Move back the pointer and ring finger -.0625 and move the pinky back -0.125 ; (7) Move the pinky joint edges back -.0625 and move the middle finger joint edges up +.0625; (8) Remove the back edges and vertices; (9) Move the wrist edges inwards by +.1 ; (10) Save file as "hand".

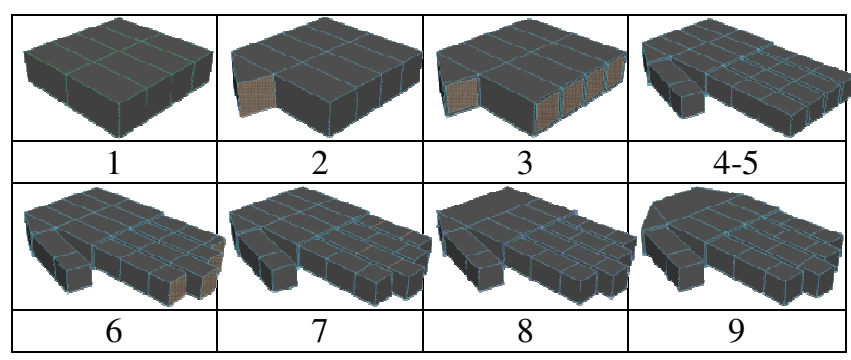

Figure 4. Modeling the Hand (see text for explanation)

\section{Part 3. Creating the Feet}

To create a foot: (1) Create a cube that is $.5 \times .25 \times 1$, with 1x2x2 faces; (2) Merge the toe vertices down; (3) Move the ankle sides in by -.1, and move the front ankle edge loop back by -.2 ; (4) Move the toes forward by +1.25 ; (5) Save file as "foot". 


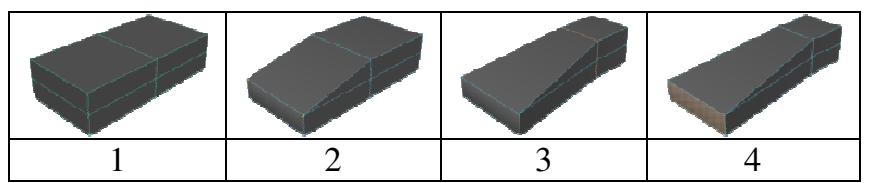

Figure 5. Modeling the Foot (see text for explanation)

\section{Part 4. Creating the Head}

To create a head, simply create a cube that is $.7 \times 1 \times 1$ with $2 \times 3 \times 3$ faces, then save the file as "head". The head can be shaped after it is attached to the body in part 5.

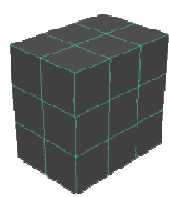

Figure 6. Modeling the Head (see text for explanation)

\section{Part 5. Combining the Pieces}

The final step is to merge all the pieces into one. After saving all the separate body parts: (1) Import the halfbody; (2) Import the foot; (3) Snap foot to leg; (4) Merge objects \& vertices; (5) Import hand; (6) Snap hand to upper limb; (7) Merge objects and vertices; (8) Mirror to get the right side of the body; (9) Attach the head; (10) Smooth the avatar if desired; (11) Save manikin.

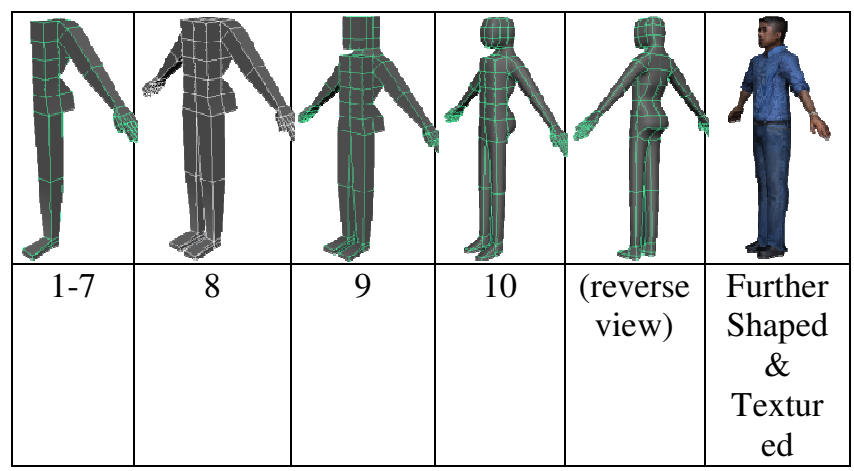

Figure 7. Attaching the Body Parts (see text for explanation)

\section{MANIKIN METHOD TEST}

\section{Participants}

Sixty-seven students participated in this study. The students came from two different courses offered in the Fall of 2010: (1) MGMT 330-Fundamentals of Virtual Business Programming; and (2) IFDM 205-Activating Digital Space. There were 43 students in MGMT330, none of which had any experience with figure drawing or 3-D modeling prior to taking the course, which was a required course for junior and senior level students majoring in management of information systems. IFDM 205 is a required course in the University's Interdisciplinary Film \& Digital Media program for sophomore students. IFDM is a highly selective program where students are admitted based on their combined artistic and technical expertise. There were 24 students in IFDM 205, all of them had been introduced to basic 3-D modeling in a prior course where they modeled a chair. Four of the IFDM students had taken a prior 3-D modeling course either at the University or at a community college, and one of the four had extensive modeling experience but was self-taught.

\section{Apparatus}

For hardware, students used their personal laptops or desktop computers to run the 3-D modeling software. The software used was Maya 2010, which the students downloaded for free from the Autodesk.com website (students.autodesk.com) as part of Autodesk's free software program for students and faculty. The IFDM computer lab also had Maya 2010 loaded on 22 personal computers in case students were unable to load Maya onto their personal computers. However, all 67 students reported successfully loading Maya onto their personal computers. The University's online instructional system, WebCT, held a link to YouTube tutorial videos made by the instructor on the topic of modeling 3-D characters and this video was made available to all students.

\section{Procedure}

Students were given a lecture on how to model 3-D figures based on the lecture script described previously. Instead of the term "manikin", I used the term "robot", which students are more familiar with. They were then given an assignment where they had a week both to create a personalized robot avatar and to animate a walk cycle for the robot avatar (see Figure 8 for the written instructions). 
Based on Professor Flor's Robot as taught in class:

1. Model a robot body in Maya (filename: body.mb).

2. Model a robot head in Maya (filename: head.mb).

3. Model a robot hand in Maya (filename: hand.mb).

4. Model a robot foot in Maya (filename: foot.mb).

5. Personalize your robot body parts.

6. Merge all the body parts to yield a robot avatar.

7. Animate a walk cycle for your robot avatar (filename: robot.mb).

8. Export your walking robot avatar as an fbx file (filename: robot.fbx).

Figure 8. Attaching the Body Parts (see text for explanation)

There were two instructional conditions based on the course. In condition 1 , students received the lecture through YouTube videos that were embedded in the University's online instructional delivery system, WebCT. The total time for the video lectures was approximately one hour. All the students in MGMT330 were in condition 1, as MGMT 330 was a purely online course with no face-to-face component. In condition 2 , students were given a one hour face-to-face lecture on modeling a humanoid figure in 3-D, and also provided access to the video lectures on WebCT for reference purposes. All the students in IFDM 205 were in condition 2.

\section{Results}

There were 24 students in IFDM 205, 22 turned in a robot, and 2 did not turn in a robot. Of the 2 that did not turn in a robot, 1 did not turn in any assignments at all. Of the 22 that turned in a robot, one person saved the file incorrectly and turned in an animated skeleton without the robot-however, upon inspection, the student did have the robot modeled. If the person that did not turn in any assignments is removed from the total number of students, $22 / 23$ or $95.6 \%$ of the students successfully turned in a robot.

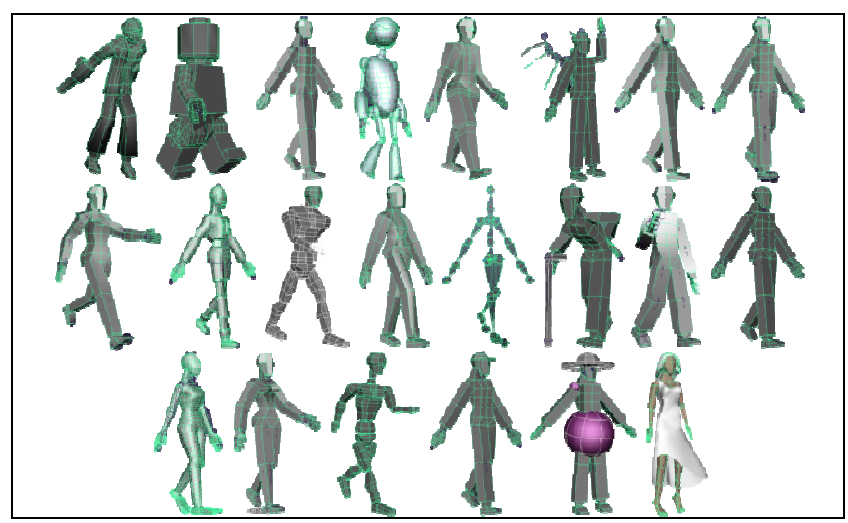

Figure 9. Robot Manikins Created by the Interdisciplinary Film \& Digital Media (IFDM 205) Students
Students made the following customizations. For the head, students added horns (1-6), hats (2-9, 2-10), and hair (2-11). For the arms and legs, students added limb \& muscle tapering $(1-5,1-10,2-6,2-7,2-11)$ and props (2$3)$. No hands were customized to any visually apparent degree. However, but two student substituted a feet with toes $(2-6,2-11)$ for the standard manikin feet. For the torso, students tapered the waist $(1-5,1-10,2-6,2-7)$, added wings (2-5), sculpted female chests (2-6, 2-7), made chest depressions (1-10); and added shoulder and waist props (2-10). Finally, two students used the automatic smoothing function in Maya to create a more rounded manikin $(2-6,2-11)$, and one student added clothes (2-11). Note: Manikins 1-1, 1-2, 1-4, 1-11, and 1-8-although highly customized-did not follow the manikin procedure. These manikins were created by students who came up with their own procedures $(1-1,1-$ $2,1-4)$, or were created in another modeling class (1-11, $2-8$ - - a fact that was only discovered several weeks after the assignment was due.

There were 43 students in MGMT330. Of these 43 students, 33 turned in a manikin, while 10 students did not turn in a manikin. Of the 10 students that did not turn in a manikin, 7 did not turn in any assignments at all during the semester. Of the 33 students that turned in a manikin, 3 did not save their files properly and only turned in an animated skeleton. However, when their work was checked in person, they indeed modeled a manikin correctly. If you removed, the 7 students that did not show up to class, then $91.6 \%$ of the students successfully followed the procedure for modeling a manikin.

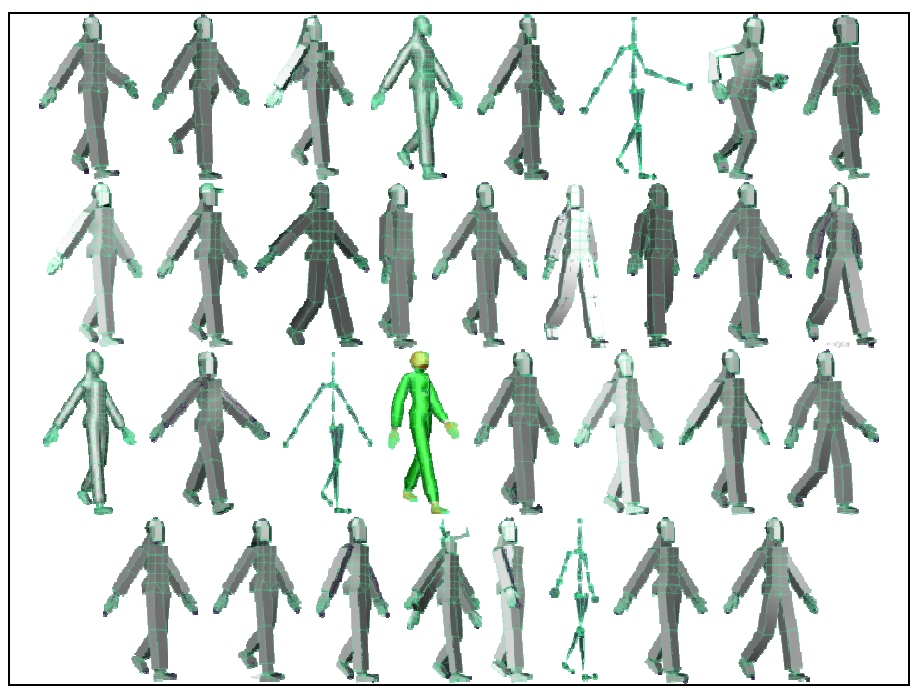

Figure 10. Robot Manikins Created by the Management (MGMT 330) Students

MGMT 330 students made the following customizations. For the head, students added hair (1-3), a hat (2-1), horns (4-5), and one substituted a pumpkin (3-5) for the manikin head from the procedure. For the arms and legs, one 
student added arm tapering (row 1-col 8), another student added multiple arms (4-5). No hands were customized to any visually apparent degree. However, one student substituted feet with heels (1-3) and another substituted flatter feet for the standard manikin feet. There was only one torso customization, where a student sculpted a female chest (1-3). Finally, three students used the automatic smoothing function in Maya to create a more rounded manikin (1-4, 3-2, and 3-5), and one student used the coloring features of Maya to create a green and orange manikin (3-5).

\section{SUMMARY AND CONCLUSION}

The ability to model human figures is a difficult skill for non-artists to acquire, using current methods like 3-D tracing or digital sculpting. The reason for the difficulty is that these methods are aimed at creating highly-detailed figures for movies and games and require either in-depth artistic knowledge or anatomical knowledge. Without this knowledge, one must collaborate with others that possess it.

The inability to model human figures in 3-D is one key deterrent for non-artists that would like to experiment with new forms of virtual worlds. This paper presented a flexible and repeatable method that non-artists can master in a short amount of time, to quickly develop 3-D human figures that are suitable for virtual world prototyping. While these figures-manikins - are not as detailed as those created by 3-D tracing or digital sculpting, they do not have to be for the purpose of experimenting with virtual worlds. However, the method is flexible enough that once one settles on a virtual world design, the details can be added later using either 3-D tracing or digital sculpting.

I presented a test of the method on two classes of students-both artists (film \& digital media students) and non-artists (management students)—with over $90 \%$ of the students in both classes able to successfully model a 3-D figure in under a week. While this is a good result and shows the repeatability of the method, the amount of customization in the student manikins was minimal. Since the manikins of the artistic students also lacked extensive customizations, I attribute the minimal customizations to a lack of emphasis in the assignment instructions.

One drawback of the manikins is that they are not detailed and do not look realistic. It is possible to layer detail and realism on the manikins (see Figure 7, last panel), but this is currently a tedious process that combines 3-D tracing with texture mapping, the latter of which can be thought of as a process of painting the body of the manikin. Yet detail and realism is desirable when moving from a virtual world prototype to a commercial virtual world product.

Future research is needed to develop methods, similar to the manikin method, that allows non-artists to quickly transform manikins into more realistic-looking human figures. For those realistic figures that are not based on actual people, this will require discovering how to teach a streamlined form of human anatomy. For figures based on actual people, an alternative to importing pictures and tracing must be discovered. I believe a method similar to drawing caricatures might suffice, where prominent features in a person are emphasized but not duplicated.

In closing, the most innovative and useful forms of virtual worlds have yet to be discovered. The manikin method, combined with knowledge of programming, animation, and terrain modeling, gives non-artists the ability to experiment with developing new forms of virtual worlds. This research is a step towards helping researchers, students, and entrepreneurs explore the space of possible virtual worlds.

\section{References}

[1] Bainbridge, W. (2007). Scientific Research Potential of Virtual Worlds. Science, 317, 472-476.

[2] de la Flor, M., \& Mongeon, B. (2010). Digital Sculpting with Mudbox. Burlington, MA: Focal Press.

[3] Dede, C. (1996). The Evolution of Constructivist Learning Environments: Immersion in Distributed, Virtual Worlds. In B. G. Wilson, Constructivist Learning Environments (pp. 165-175). Englewood Cliffs, New Jersey: Educational Technologies Publications.

[4] Flor, N. V. (2011). Self-Developing a MUVE for Research and Educational Innovations. In G. Vincenti , \& J. Braman , Teaching through Multi-User Virtual Environments: Applying Dynamic Elements to the Modern Classroom (pp. 453-470). Hershey, PA: IGI Global.

[5] Guindon, M.-A. (2007). Learning Autodesk Maya 2008: The Modeling and Animation Handbook. San Rafael, CA: Autodesk, Inc.

[6] Hemp, P. (2006). Avatar-Based Modeling. Harvard Business Review, June, 1-8.

[7] Hevner, A. R., March, S. T., Park, J., \& Ram, S. (2004). Design Science in Information Systems Research. MIS Quarterly, 75-105.

[8] Ingrassia, M. (2009). Maya for Games. Burlington: Elsevier.

[9] Loomis, A. (1943). Figure Drawing for All It's Worth. New York: Viking Press.

[10] Maglio, P. P., \& Spohrer, J. (2008). Fundamentals of Service Science. Journal of the Academy of Marketing Science, 36, 18-20.

[11] Oliverio, G. (2007). Maya 8: Character Modeling. Plano: Wordware Publishing.

[12] Olson. (2009, July 24). Interview With 3D Character Artist from Blitz Game Studios Jose Lazaro. Retrieved January 7, 2012, from TutorialBoard.Net: http://www.tutorialboard.net/interview-3d-character-artistblitz-game-studios-jose-lazaro/

[13] Patnode, J. (2008). Character Modeling with Maya and ZBrush. Burlington, MA: Focal Press.

[14] Ratner, P. (2009). 3-D Human Modeling and Animation. Hoboken, New Jersey: Wiley \& Sons.

[15] Russo, M. (2006). Polygon Modeling: Basic and Advanced Techniques. Plano: Wordware Publishing.

[16] Simon, H. (1996). The Sciences of the Artificial. Cambridge, MA: MIT Press.

[17] Spencer, S. (2010). ZBrush Digital Sculpting: Human Anatomy. Indianapolis: Wiley Publishing. 
[18] Vincenti, G., \& Braman, J. (2010). Teaching Through Multi-User Virtual Environments: Applying Dynamic Elements in the Modern Classroom. Hershey, PA: IGI Global.

\section{APPENDIX A: TUTORIAL-BASIC POLYGON MODELING OPERATIONS IN MAYA}

The following tutorial describes the basic polygonmodeling operations used by the Manikin Method to model a character in Maya, which can be obtained freely at students.autodesk.com for non-commercial, academic use only. While Maya is just one of many 3-D modeling software packages available, almost all modeling software supports the operations that will be described-the differences lie in the user-interfaces. If you are unfamiliar with 3-D modeling, you should first familiarize yourself with these basic operations before attempting the Manikin Method.

\section{Enter Polygon Modeling Mode <F3>}

The following operations assume you are in Maya's polygon-modeling mode. If you are not in this mode, the proper menus will not be displayed. The shortcut for entering polygon mode is the $\langle\mathrm{F} 3\rangle$ key. There is also a drop-down box in the upper-right corner of the program, but this drop-down is not always displayed unless you select the menu item Display > UI Elements $>$ Show All UI Elements.

\section{Create Box Operation}

In polygon modeling, you use boxes with various dimensions and divisions as the starting point for different body parts. Suppose you wanted to create a box that represents the torso of a human figure. Further, assume you want the box to have a width $\mathrm{x}$ height $\mathrm{x}$ depth of $1 \mathrm{x}$ $2.5 \times 1$, and to have edge-loop divisions of $2 \times 5 \times 2$.

The steps to create this box are as follows (refer to Error! Reference source not found., italicized text refers to labels in the Maya user interface. Unless otherwise stated, a click or select action refers to a left-mouse click.):

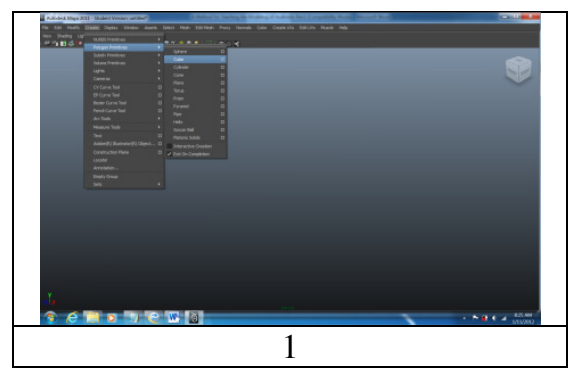

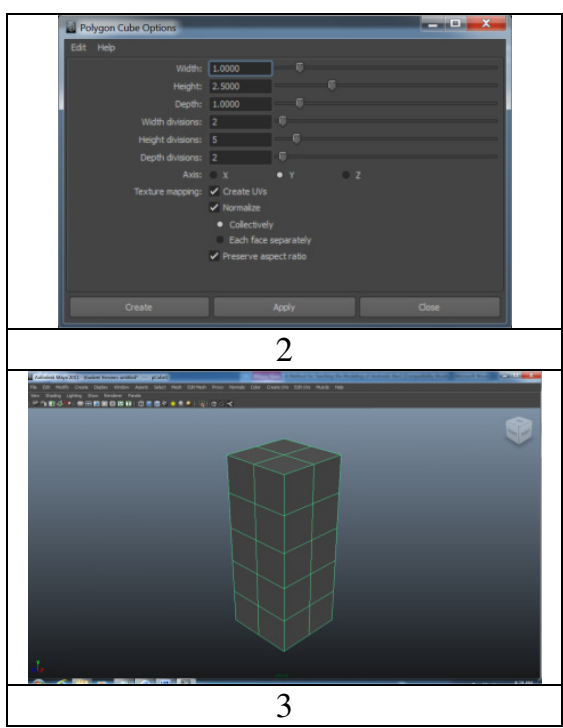

Figure 11. Robot Manikins Created by the Management (MGMT 330) Students

1. Select the menu item: Create $>$ Polygon Primitives $>$ Cube $>$ Options (options are selected by clicking the square next to the label).

a. Note: Ensure that the Interactive Creation box is unchecked.

b. Response: The Polygon Cube Options window opens.

2. Enter 1, 2.5, and 1 in the Width, Height, and Depth textboxes. Enter 2, 5, 2 in the Width divisions, Height divisions, and Depth divisions textboxes, then click the Create button.

a. Response: A box is created in the workspace.

3. (A box is displayed with dimensions $1 \times 2.5 \times 1$, and $2 \times 5 \times 2$ edge loop divisions)

\section{Wedge Operation}

Wedges are useful for creating body parts that are angled. Examples include the base of the thumb relative to the palm of the hand, and the shoulder relative to the torso. The following shows how to create a 45 degree wedge that represents a shoulder on a torso (refer to Figure 12):

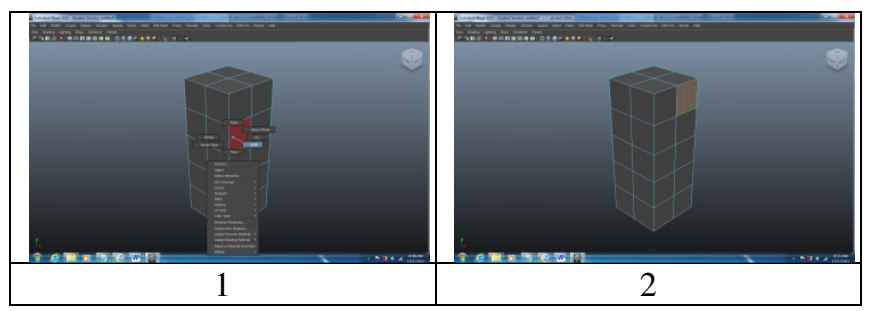




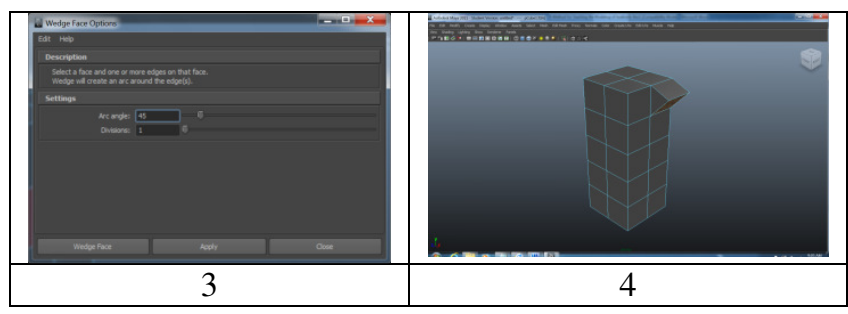

Figure 12. Wedge operation

1. Right-click anywhere over the object and select Multi.

a. Explanation: This is a selection mode that allows you to choose groups of faces, edges, or vertices.

b. Response: None visible. You are in the Multi selection mode.

2. Click on the face that is furthest back, on the top-rightside of the cube. Shift-click on the bottom edge of this face.

a. Explanation: The wedge operation requires both a face and an edge, where the edge is like a hinge that the face swings around to create the wedge.

b. Response: The select face and the edge are highlighted.

3. Select the menu item: Edit Mesh > Wedge Face > Options

a. Response: The Wedge Face Options window opens.

4. Enter 45 and 1 into the Arc angle: and Divisions: textboxes, respectively. Then click the Wedge Face button.

a. Explanation: The 45 is the wedge's sweep angle in degrees, and the 1 determines how many slices are in the wedge.

b. Response: A wedge of 45 degrees.

\section{Extrude Operation \& A Note on the Inset Operation}

The extrude operation is useful for modeling limbs, fingers, and other protrusion on the human body. The following example uses the extrude operation to create an arm (refer to Figure 13):

1. Click on the shoulder face.

a. Note: You should be in face-selection mode, via the menu item Select $>$ Face

b. Response: The shoulder face is highlighted.

2. Select the menu item Edit Mesh $>$ Extrude $>$ Options

a. Response: The Extrude Face Options window opens.

3. Enter 2 into the Divisions: textbox, and enter . 1 into the Offset: textbox, then click the Extrude button.

a. Note: Leave all other values at their defaults.

b. Explanation: When you extrude, the default is a single extrusion. Entering 2 divides the extrusion into two pieces, representing the upper and lower arms. The offset controls the size of the extrusion's ending stump. The .1 moves all edges on the stump inwards by .1 units. This will ultimately lead to a skinny wrist relative to the upper and lower arms.

c. Response : Control arrows for pulling out the extrusion on the shoulder face.
4. (Extrusion control arrows displayed over the face)

a. Note: At this point, you could pull the arrow that is normal to the face in order to create an arm.

However, according to artistic canons of proportion, the arm must be a specific length, namely 2.5 units. The next step allows you enter a specific length for an extrusion.

5. Select the menu item: Display $>$ UI Elements $>$ Attribute Editor.

a. Response: The Attribute Editor pane appears on the right side of the workspace.

6. Type 2.5 into the Local Translate $Z$ textbox then press $<$ Enter $>$. The $\mathrm{Z}$ textbox is the third textbox to the right of the label, and always corresponds to the control arrow that is normal to the face.

a. Response: An extrusion representing an upper arm, lower arm, and wrist

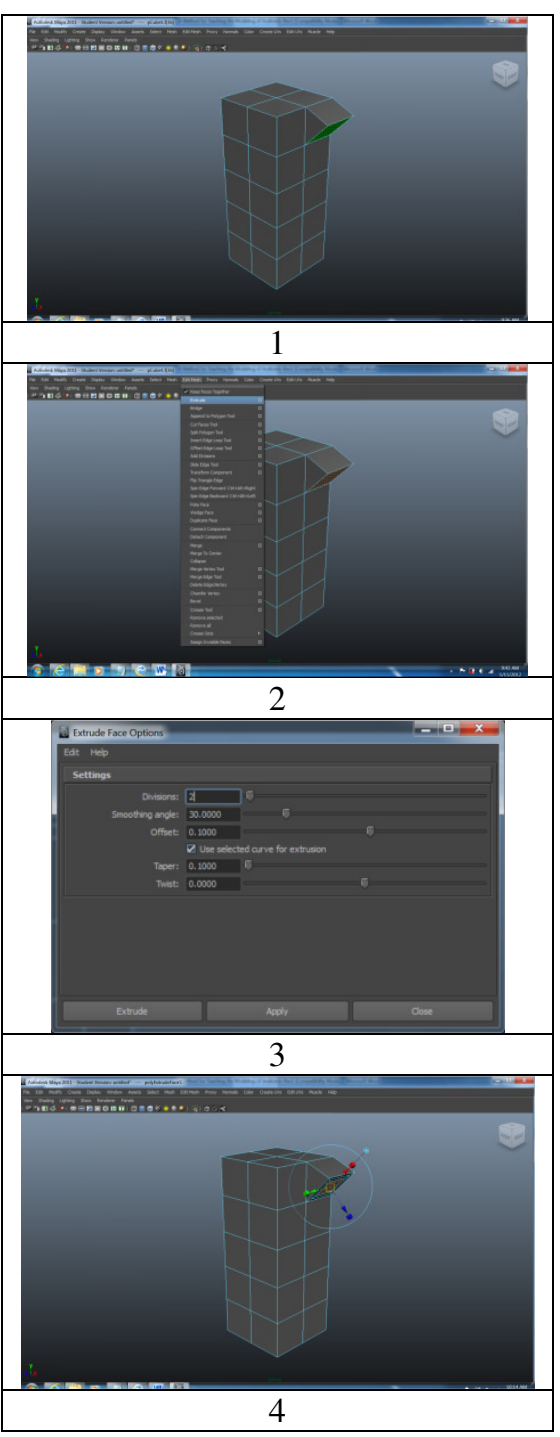




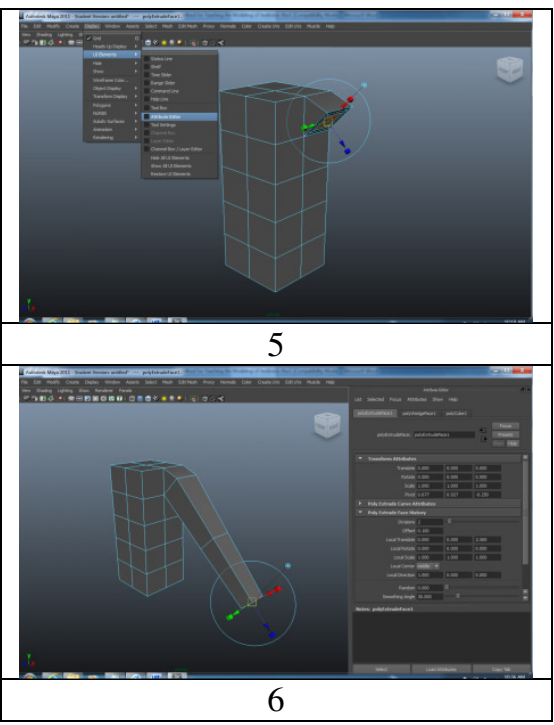

Figure 13. Extrude operation

\section{A Brief Note on the Inset Operation}

A face within a face is known as an "inset". You create an inset by doing the extrude operation with an offset (Figure 13, steps 1-4), but not pulling out an extrusion (viz., not doings steps 5-6 in Figure 13). Figure 14 depicts a close-up of the inset created by Steps 1-4.

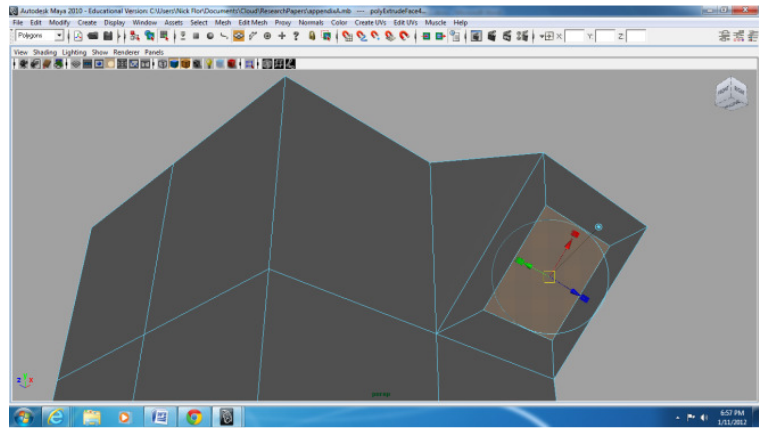

Figure 14. A Close-Up of an Inset

\section{Moving Elements-Relative Movement \& a Note on Absolute Movement}

To move a vertex, an edge, or a face of an object freehand, you: (a) enter either vertex mode , edge mode, or face mode, via the menu items Select $>$ Vertex, Select $>$ Edge, or Select > Face; (b) select a vertex, edge, or face; (c) select the move icon in the toolbox bar; (c) click and drag the vertex, edge, or face. However, moving elements freehand is not precise. The following demonstrates how to move vertices in a relative direction, by a specific amount, +0.1 in the $\mathrm{X}$-direction, to prepare a face for a leg extrusion.

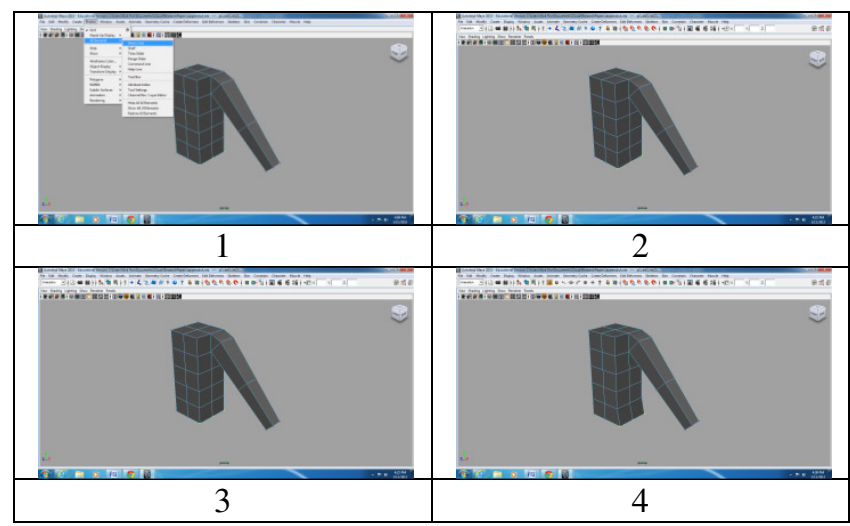

Figure 15. Move Relative operation

1. If the user-interface Status Line is not displayed, display it by selecting the menu item Display $>$ UI Elements > Shelf.

a. Explanation: The Status Line is a toolbar that contains 3 textboxes known as the "Input Line", which allows you to type in $\mathrm{X}-\mathrm{Y}-\mathrm{Z}$ values for vertices.

b. Response: The Status Line toolbar appears

2. Select a vertex or shift-select multiple vertices, which are to be moved.

a. Note: I selected the lowermost three vertices on the right side of the torso.

b. Response: The selected vertices are highlighted.

3. Select Relative transform in the drop-down box to the left of the Input Line.

a. Response: icon image changes to the relative indicator.

4. Enter .1 into the X: textbox of the Input Line then press $<$ Enter $>$.

a. Response: the bottommost, right-side vertices move to the right .1 units.

\section{A Brief Note on Moving Elements in Absolute Mode}

To move elements to absolute positions, in step 3 do a select Absolute transform instead of a Relative transform, all the other steps are the same. Figure depicts the fourth row of back vertices moved to $\mathrm{Z}=0$, to create a back wedge.

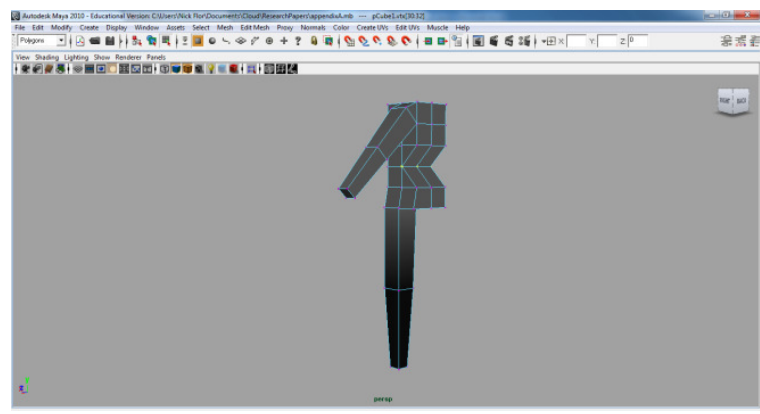

Figure 16. The Fourth Row of Back Vertices Moved Absolute to $Z=0$, to Create a Back Wedge 


\section{Merge Operation-Relative \& a Note on Absolute Movement}

The merge operation takes two or more vertices either that are close to one another, or that overlap one another, and makes them into a single vertex. Merge is useful for connecting separate body parts, such as a hand to an arm, or a foot to a leg, or a head to a torso. Note: before merging separate models, they must be combined via the menu item Mesh > Combine.

Moreover, when modeling a human figure using the Basic Manikin Method in this paper, there are situations where you will move and overlap vertices. Examples include creating a back wedge (see Figure 16), or making a box into a foot (see Figure 5, Step 2). By applying the merge operation to the overlapped vertices, you remove the extraneous points.

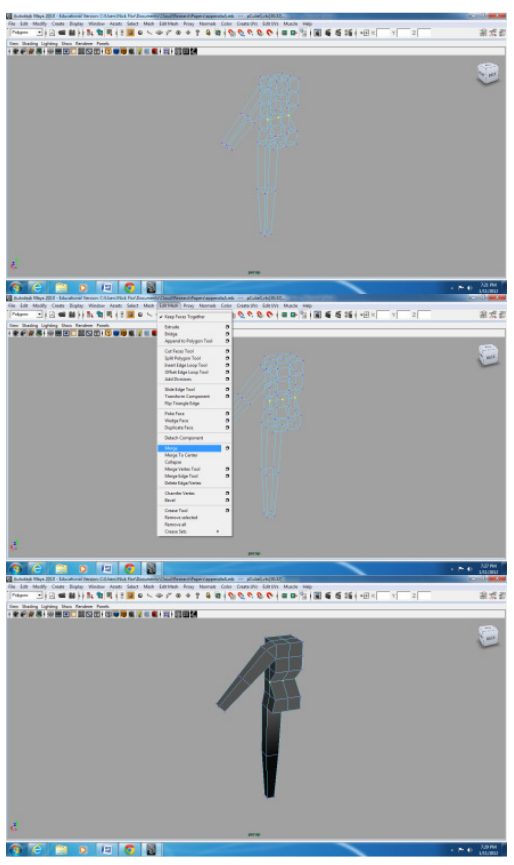

Figure 17. Merge Operation Applied to the Back Wedge Vertices

To merge vertices:

1. Drag-select the vertices that overlap, by using the mouse to drag a rectangle that encloses the overlapping vertices.

a. Note: It is best to select overlapping vertices in wireframe mode, which can be entered by pressing the $<4>$ key as a shortcut, or via the workspace menu item Shading $>$ Wireframe.

b. Response: The overlapping vertices are highlighted.

2. Select the menu item Edit Mesh > Merge.

a. Response: There is no visible response, but any overlapping vertices become a single vertex.

3. (The model with the back vertices merged)

\section{Mirror Operation}

Modelers use the mirror operation to save time. Mirroring is appropriate whenever the object to be modeled is largely symmetrical, such as a body or a head. To mirror, a modeler creates half the object, and uses the mirror operation to create the other half automatically via the software.

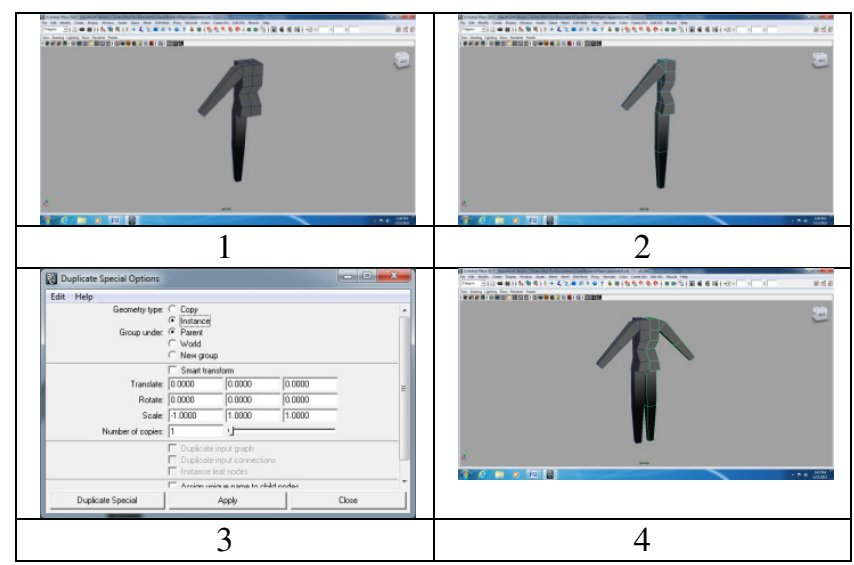

Figure 18. An Example of the Mirror Operation

The steps to mirror an object are:

1. (Model half of an object)

2. Delete any faces on the other half of the object by first selecting the menu item Select $>$ Face, then dragselecting the unmodeled half of the object, and finally pressing the <delete $>$ key.

a. Response: Half of the object is displayed.

3. Mirror the other half of the body by first selecting the menu item Select $>$ Object / Component, selecting the object, then selecting the menu item Edit $>$ Duplicate Special > Options. When the Duplicate Special Options window opens: (a) select the Instance radio button; (b) type -1 into the first Scale: textbox; and (c) press the Duplicate Special button.

a. Explanation: Typing -1 into the very first Scale: textbox, mirrors the object across the $\mathrm{X}$-axis by taking every vertex in the object and making a copy of it except with the X-coordinate negated.

b. Response: The other half of the object is automatically created.

4. (The result of the mirror operation) 